\title{
Community-directed treatment of lymphatic filariasis in Kenya and its role in the national programmes for elimination of lymphatic filariasis
}

\author{
Njeri Wamae*, Sammy M. Njenga, Wilfred M. Kisingu, Pauline W. Muthigani and Karanja Kiiru \\ Kenya Medical Research Institute (KEMRI), P.O. Box 54840-00200, Nairobi, Kenya \\ *Author for Correspondence. Tel : 254-2-02720409 ; Fax: 254-2-02737658; e-mail: nwamae@kemri- \\ nuitm.or.ke
}

\begin{abstract}
SUMMARY
We conducted a prospective, cross-sectional study to examine and compare treatment coverage of lymphatic filariasis by the health system (HST) and a health system implemented, community-directed treatment for the control of lymphatic filariasis (ComDT/HS) in 44 randomly selected villages in coastal Kenya. Demographic information on the villages and peripheral health facilities to guide design and implementation was obtained from a situation analysis phase of this study. A series of interactive training sessions on basic biology of lymphatic filariasis, concept and philosophy of ComDT/HS were given to members of the District Health Management Team (DHMT), peripheral health staff, community leaders and community drug distributors (CDDs) prior to ivermectin distribution. An intensive sensitization process of the community by the trained peripheral health staff and community leaders followed before selection of the CDDs. Quantitative and qualitative data for evaluation of the study were collected by coverage surveys of randomly selected households, focus group discussions and interviews, immediately after the drug distribution. Treatment coverage of all eligible persons was 46.5 and $88 \%$ in HST and ComDT/HS villages, respectively, $\mathrm{P}<0.001$. In comparing treatment coverage by the two study arms in relationship to the distance from a health facility, coverage among HST and not ComDT/HS villages was influenced by distance. In Kenya, ComDT/HS can effectively be implemented by the regular health system and can attain coverage levels compatible with the global filariasis elimination goal.
\end{abstract}

[Afr J Health Sci. 2006; 13: 69-79]

\section{Introduction}

The introduction of single-dose treatment regimens of either diethylcarbamazine (DEC) or ivermectin with albendazole [1] has been an important breakthrough for filariasis control. The global strategy for control of lymphatic filariasis has therefore, been redefined, and is now principally based on annual, single-dose treatment of all eligible members of endemic communities. The main challenge that remains for filariasis elimination is to deliver treatment to the endemic populations, and to sustain annual delivery with high treatment coverage for a sufficiently long period (4-6 years) to bring about elimination of the disease. In most endemic countries, sustained drug delivery to all high-risk communities is difficult to achieve by the health services alone, either because they are overburdened with other responsibilities and/or short of resources. Additionally, lack of active participation of the population with official health treatment programmes may also be a drawback. Recent research on drug delivery for another filarial disease, onchocerciasis, indicates that greater involvement of the endemic 


\section{RESEARCH ARTICLE}

communities in the delivery process may be a solution [2]. This large, multi-country study has shown that community-directed treatment of onchocerciasis is feasible and effective in onchocerciasis control; and is the basis for the control strategy of the African Programme for Onchocerciasis Control (APOC) [3].

Onchocerciasis was previously eradicated from Kenya [4] and unlike other neighbouring APOC countries, such as, the Sudan, Tanzania, and Uganda; Kenya has no similar experience with community-directed treatment. Lymphatic filariasis in Kenya has been reported since 1910 [5] and the disease remains endemic in the six districts of Kwale, Mombasa, Malindi, Kilifi, Tana River and Lamu in coastal Kenya where about 2.5 million people [6] are at risk of infection. Recent epidemiological studies report microfilaraemia prevalence in these areas as 15$25 \%$ [7-10] and antigenaemia as 35\% and above[7,10]. Additionally, the situation in the Lake Victoria Region remains unclear but there is strong speculation for presence of lymphatic filariasis in the region. In preparation for the ongoing Global Programme to Eliminate Lymphatic Filariasis (GPELF), we participated in a two phase, multi-country study of community-directed treatment of lymphatic filariasis between July 1997 and October 1999. Other participating countries were Ghana, India, Myanmar and Vietnam. The purpose of the study was to compare the effectiveness of a drug delivery strategy based on mass-treatment by the regular health service (HST) with that of community-directed with health system involvement at the implementation stage only (ComDT/HS). The findings from the Kenyan site and their implications for both Kenya's National Programme for Elimination of Lymphatic Filariasis (NPELF) and GPELF are discussed. Ethical approval to conduct this study was given by the Kenya Medical Research Institute's Ethical Review Committee.

\section{Study Design and Methods Study area}

The study area covered both Kilifi and Malindi districts, two of the six districts in coastal Kenya (Fig 1). The selection of these two districts was based on their known endemicity of filariasis as revealed by historical data and recent research findings including those of Phase I of the current study. The communities are rural and mostly engage in subsistence agricultural and fishing activities. The study units were half districts, thus giving a total of four implementation units for both districts. The southern part of each district was arbitrarily assigned to the HST arm and the northern to the ComDT/HS arm (Fig 1).

\section{Situation analysis (Phase 1)}

Prior to implementing the study, we conducted a situation analysis study-Phase 1 [11]. Basic data on the socio-economic situation, knowledge and perception of filariasis, presence and performance of health services and other information relevant to the design of the implementation phase, were collected. Briefly, the framework of the district health system in Kenya has three broad levels namely, the district hospital, the health centers and the dispensaries. In both the study sites there was a total of 2 district hospitals, 6 health centers and 34 dispensaries. Health facilities in both the study districts were few compared to the population $(830,000)$ they were expected to serve. A shortage of health workers to man these health facilities was observed in both districts. In communities without health facilities, residents had to walk long distances of up to $19 \mathrm{Km}$ to the health centers or dispensaries. Being the lowest level of the health system's interaction with the communities, we chose the dispensary for implementation of this study.

At the village level, there are village health committees whose responsibility is to oversee the interests of the local community in the local health facility. Their duties include general maintenance of the health facility, fund raising, administration of the cost-sharing scheme, and sometimes, collection of supplies from the district level. A total of 360 households were sampled from each of the two districts. Of 720 persons interviewed, 99\% reported that they knew about elephantiasis and hydrocele and it was reported that there existed well-defined terminologies, "matende" and, "mshipa wa kutserera", respectively. However, only 234 


\section{RESEARCH ARTICLE}

individuals associated elephantiasis to mosquitoes. Thus, $67.5 \%$ had no knowledge about this association and attributed the disease to heredity $(14.4 \%)$, fate/destiny $(10 \%)$, evil spirits $(1.7 \%)$, collection of "bad fluid" in the body and fever $(5.7 \%)$ and the remainder associated it to various diets. With regard to the burden of the disease, it was clearly indicated that the disease was an impediment to carrying out normal activities (marriage, leadership roles) and $76 \%$ knew that the disease could be treated or prevented by modern medicine. According to the health workers interviewed, the community members were not to be expected to distribute drugs independently. The health workers felt that the health staff should be involved in some way as problems due to lack of adequate knowledge on how to handle drugs, risk of overdosing the minors and loss of credibility of the programme by the community due to lack of non-medical persons would make the programme fail. Selection of study villages

We selected a total of 40 villages from the four implementing units. Eight of the villages had a dispensary within the village (dispensary villages) while the other 32 villages had the dispensary located 5 or more Km away (nondispensary villages). The 40 villages were equally assigned to either ComDT/HS or HST arm. In addition to the 40 villages selected above, four more villages were randomly selected for the ComDT/HS, Research Team (RT)-arm to serve as reference standards for evaluation. The process of selection of ComDT/HS, RT-arm was the same in principle as the one used to select HST and ComDT/HS villages. Therefore, a total of 44 villages from the two districts were selected for this study phase (Fig 1). In the reference villages, both the health staff and the research team introduced the ComDT/HS concept. In the current study, ivermectin tablets were obtained from WHO/TDR and received by the Principal Investigator who delivered them to the District Pharmacist at the district level for onward dispatch to the dispensaries. In addition to the ivermectin tablets, the study issued drugs for management of side effects. Other activities related to the implementation of ivermectin delivery in the various arms, are listed in Table 1 .

Table 1. Activities related to implementation of drug distribution by drug distribution method, ComDT Study: Kenya, 1999

\begin{tabular}{|c|c|c|}
\hline Activities & HST & ComDT/HS \\
\hline Training of key trainers & Senior health officials & Senior health officials \\
\hline Training of implementation staff & Key trainers & Key trainers \\
\hline IEC campaign & DHMT/health worker & DHMT/health worker \\
\hline Sensitisation of community & Health worker & Health worker \\
\hline Selection of CDDs & & Community \\
\hline Training of CDDs & & DHMT/health worker \\
\hline Selection of drug delivery method & Health worker & Community \\
\hline $\begin{array}{l}\text { Decision on timetable for drug } \\
\text { delivery }\end{array}$ & DHMT & Community \\
\hline Drug distribution & Health worker & CDDs \\
\hline Record Keeping & Health worker & CDDs \\
\hline
\end{tabular}




\section{RESEARCH ARTICLE}

\section{Sensitisation of the official health system to} the concept of ComDT/HS and training

Prior to the implementation of the drug distribution, the RT conducted sensitization meetings with the entire health system. Among issues discussed include, the APOC experiences with community drug distribution, the resolution by the World Health Organisation to have endemic member states eliminate lymphatic filariasis (World Health Assembly, Resolution 50.29), and our findings from Phase I. Additionally, the concept and philosophy of ComDT was introduced and the plans for its implementation explained. The RT conducted interactive training for the DHMT. The training included lectures and plenary discussions. At the conclusion of the DHMT training, one member was nominated to become the District ComDT Coordinator (DCC) and act as the liaison officer between the health workers at the dispensary level, the RT and the DHMT. Subsequently, members of the DHMT conducted a series of training for peripheral health staff, community leaders and CDDs.

\section{Sensitisation of the community and selection of CDDs}

At the conclusion of the training program, the trained peripheral health staff and community leaders went back to their respective villages and through "barazas" (public meetings) convened by the official administration, sensitized the communities on ComDT. Both posters and leaflets were used for community mobilization and sensitization campaigns. Some posters were posted at the trading centers, schools and dispensaries while leaflets were used at the dispensaries by the health workers as references.

While "barazas" were the main forum for sensitizing and mobilizing the communities, the trained community leaders also used mosques and churches for delivering the ComDT messages. School children were also sensitized at school assemblies and used as a medium for taking the ComDT messages back home. Furthermore, the peripheral health staff also used "health talks" for conveying ComDT messages to persons presenting at the dispensaries and the Public Health Technicians delivered the ComDT messages to the communities during their routine community visits. The community did the selection of CDDs at the "barazas". Two CDDs were selected from each ComDT village and attended the CDD training as described above.

\section{Record keeping}

A standard method of record keeping for both arms of the study was agreed upon. Each person's height, as surrogate for weight was measured using a standard measuring stick provided by the Project. The stick was graded in centimeters: $90-119 \mathrm{~cm}, 1$ tablet; $120-140 \mathrm{~cm}, 2$ tablets; $141-158 \mathrm{~cm}, 3$ tablets; $158 \mathrm{~cm}$ and above, 4 tablets. Record keeping stationery was also provided to both arms by the Project. Additionally, the ComDT/HS arm was provided with referral cards for filling in the identification details and remarks on any side effects that the CDD may not manage for referral to the dispensary.

\section{Evaluation}

Evaluation was based on a total of 752 house coverage surveys, including 817 semi-structured interviews (SSI) with household heads, 65 SSI with drug distributors, health workers and key informants; and 14 focus group discussions (FGD). The drug distribution period varied from two to four weeks and evaluation followed soon thereafter. All 44 villages in the study were selected for the final household coverage survey. In each village, 20 households were selected and the head of the household or a designated adult member, who was present during the drug delivery, was the interviewee. Additionally, 12 villages were randomly selected for qualitative data collection from among the 44 villages. This was for comparison of the process and implementation of the drug distribution strategies by the two methods.

Quantitative data were collected by administration of questionnaires in the coverage survey and analyzed by SPSS program. Qualitative data were coded and analyzed by Textbase Beta software. All tools for data collection had previously been developed, tested and validated during previous ComDT multi- 


\section{RESEARCH ARTICLE}

country workshops. Since both quantitative and qualitative results of the ComDT/HS, RT-arm, the reference standard, did not differ from those of ComDT/HS, they have been reported along with and as ComDT/HS findings. All quotes reported in this study are those captured by the evaluation tools with high frequency.

\section{Results}

\section{Drug distribution and treatment coverage}

In the HST arm, the peripheral health staff distributed the drugs through a central point such as the dispensary or the school, while in the ComDT/HS arm the CDDs distributed the drugs mainly on a house-to-house basis. In very few cases, the CDDs asked persons who were difficult to catch at their homes to assemble on a given date at some central location in order to receive their drugs. Both arms supervised the swallowing to ensure that those who got the drugs actually swallowed them. The coverage survey questionnaire was administered to 752 sample households and overall, a total of 3,465 people were interviewed. Female respondents were slightly more $(51.4 \%)$ than male respondents but the mean age was the same for both sexes. Respondents on average were 27.2 years. We defined treatment coverage as the percentage of respondents who reported to have received and swallowed the tablets among the 3,465 people interviewed. With respect to the distribution method, treatment coverage was 46.5 and $88 \%$ by the HST and ComDT/HS arm, respectively ( $\mathrm{P}<0.001$; Table 2$)$. Coverage was the same for males and females, and there was not much variation by age. Of the surveyed population not treated, those under the HST were more $(53.5 \%)$ than those under the ComDT/HS arm (12.0\%) and the difference was significant $(\mathrm{P}<0.001)$.

Table 2.Treatment coverage by drug distribution method, ComDT Study: Kenya, 1999

\begin{tabular}{lccc} 
Distribution method & Treated & Not treated & Total \\
\hline \multirow{2}{*}{ HST } & 799 & & \\
& $(46.5 \%)$ & $(53.5 \%)$ & $(100 \%)$ \\
Com-DT/HS & 1537 & 210 & 1734 \\
& $(88 \%)$ & $(12.0 \%)$ & $(100 \%)$ \\
Total & 2336 & 1129 & 3465 \\
& $(67.4 \%)$ & $(32.6 \%)$ & $(100 \%)$ \\
\hline
\end{tabular}

It is known that health care utilization is greatly influenced by distance to the nearest health facility. We therefore, assessed whether distance to the facility affected the results of the present study, where the health worker had to travel the same distance to provide treatment (HST) or introduce Com-DT/HS. As described in the methodology above, selection of study villages was stratified by distance to the nearest dispensary with some of the study villages located within $5 \mathrm{Km}$ of the dispensary and others at more than $5 \mathrm{Km}$ distance. As one would expect, the treatment coverage among the HST villages was much lower in the distant villages than those located close to the dispensary. However, among the Com-DT/HS villages the distance factor did not seem to affect the treatment coverage and it was similar for villages close or far from the dispensary. With respect to Com-DT/HS, this response was given:

"In fact going house to house was the best thing because you reach even those who can not walk. The people liked it because they felt it was not interfering with their daily duties, drug distributors were people they knew and they had faith in them." (Health Worker)

Almost everyone (2351) who received the drugs had swallowed them with only $0.4 \%$ 
persons claiming not to have swallowed. In the few cases where the drugs were not swallowed, reasons given for failure to swallow were not unexpected. In some cases it was due to religious affiliation or suspicion that the drugs were for contraception or that it was a government conspiracy to decimate the coastal people.

"Education is important. They also think it is for family planning. In fact, even at home there is my co-wife who never took the drugs for fear of death." (FGD)

\section{Sensitisation process, Selection of CDDs and Awareness of the drug delivery}

From the qualitative data, we received the following responses in the ComDT/HS communities with respect to sensitization:

"The health officer was going from house to house telling people there would be drugs for hydroceles and elephantiasis." (Key Informant)

It was clear that the sensitisation process had emphasized the role of the community in CDD selection:

"Yes, as I said we also trained the chiefs and they helped us by calling barazas (public meetings). We told them about the project. The Assistant chief told the Wananchi (Public) about the selection of the CDDs, who we took for training. The Wananchi are the ones who participated with the selection of the CDDs." (Health Worker)

From the house survey, some 388 out of $392(99.0 \%)$ and 308 out of $360(85.6 \%)$ respondents in Com-DT/HS and HST, respectively, reported that they were aware of the drug distribution programme. However, there was a significant difference between the HST and Com-DT/HS, with 52 respondents $(14.4 \%)$ being unaware of the drug distribution in HST compared to $4(1.0 \%)$ in Com-DT/ HS arm. Information, Education and Communication (IEC) materials also played a role in creating awareness in community members:

"Yes, I have heard about the drugs, in fact I saw the posters at the clinic door but I have not received them (drugs)." (FGD)
We also wanted to know whether the respondents were clear about the disease target that the drug was for. Although the respondents in the Com-DT/HS arm were more aware of the existence of the drug delivery, they were less clear $(16 \%)$ about its purpose than in the HST arm $(29 \%)$ and this difference was statistically significant $(\mathrm{P}<0$.001).

\section{Record keeping, side effects and their management}

The evaluation did not reveal any difference in record keeping between the two arms. Among the 752 respondents, $96 \%$ reported to have experienced no side effects. Side effects were reported more often by the Com-DT/HS than by the HST arm. They included giddiness (1.1\%), fever $(0.8 \%)$, headache $(0.3 \%)$, vomiting $(0.3 \%)$ and myalagia (1\%). Persons showing side effects in the ComDT/HS arm were issued with referral cards and referred to their village dispensary for management.

"Very few people reported headaches and dizziness, but when they took panadols, all the symptoms subsided.." (Health Worker)

\section{Discussion}

In this study, Com-DT/HS performed well and achieved high treatment coverage (88.0\%). Although it is not yet known precisely what level of treatment coverage is required to achieve global elimination of filariasis, computer simulations suggest that coverage of about $80 \%$ over a 5 years period may be sufficient to interrupt transmission [12]. Based on this criterion, it appears that Com-DT/HS in Kenya can achieve the treatment coverage required for the elimination of lymphatic filariasis. In contrast, the treatment coverage achieved by the HST arm was far below what may be expected to achieve transmission interruption.

Health-care utilization is greatly influenced by distance to the nearest health facility [13]. The distance of the community to the health facility did not seem to affect treatment coverage in the Com-DT/HS villages where it was the same for villages close or far from the dispensary. One possible explanation from the 


\section{RESEARCH ARTICLE}

qualitative data is that CDDs in the Com-DT/HS were considered more motivated to ensure the collection and distribution of the ivermectin tablets, even if some of them had to travel longer distances than the health workers.

Since the GPELF is dependent on several rounds of drug distribution, it may be argued that it is premature to conclude that the successful coverage we achieved with one experimental round would always be achieved in subsequent rounds in a large-scale situation. It is recommended therefore, that community sensitisation and training of CDDs remain continuous and intensive in order to give CDDs more confidence in what maybe viewed as a duty for the medical professionals, create a forum for feedback and addressing any hiccups that a national PELF may experience thereby, empowering the communities to gain ownership of the program. However, as much as gaining ownership of a program such as this is important, limitations of the community must be acknowledged before making unrealistic demands on them. Appreciation of the efforts required for daily subsistence may leave little capacity for rural communities to provide voluntary support to such programs [14] and hence affect the sustainability. In the present study, remuneration of the CDDs was a contentious issue and some communities devised different ways for cash and kind incentives such as using the cost-sharing funds at their health facilities to remunerate their CDDs.

In Kabarole district, Uganda initial community efforts to support ivermectin distributors for river blindness control, proved inadequate and the presence of an active HIV/AIDS programme with a condom social marketing component, provided an opportunity for the drug distributors to retail condoms. Retailing of condoms facilitated making of some income by the drug distributors, promoted sustainability of ivermectin distribution as well as addressing other important health issues [15]. The health service and other partners with ongoing community activities at national PELF sites should therefore, avail similar opportunities to the communities as an option for communitydevised CDDs' incentive mechanisms. Caution must be taken, however, to ensure that availing and implementing alternative mechanisms for CDDs' incentives does so with community participation and the ComDT philosophy is not violated. Mutabazi and Duke ${ }^{15}$ have observed low or non-participation of women in onchocerciasis treatment in Uganda and suggested that women should not only participate but also should be available for appointment as CDDs. Similarly, Askew and Khan [16], in a study of community-based delivery of family planning made a similar suggestion. In the present study women participated in both the barazas and selection of CDDs, and up to $53 \%$ of the CDDs were female.

However, in informal discussions on the composition of CDDs, the community indicated that married women were tied by the demanding domestic chores and it was unwise to invest in unmarried female CDDs as they would end up marrying outside the community and be unavailable for subsequent rounds of distribution. Therefore, as suggested in a parallel study in Ghana, communities understand the role that their community members can perform best and should be allowed to select their own CDDs [17]. Moreover, selection of CDDs by the community was found to be a useful indicator for predicting sustainability and monitoring progress towards self-sustenance of drug delivery in Uganda [18]. Nearly everybody who received the drugs swallowed them, and compliance with treatment was not a problem. During the sensitizations, the importance of community versus personal gains from the treatment was emphasized and under no circumstances were drugs for absent members left with the community for onward delivery.

The high compliance may therefore be attributed to the drug swallowing observation approach adopted by both arms. To sustain such high compliance in any national PELF, the same approach is recommended. There were few cases of persons who adopted a "wait and see attitude" but on realizing that those who complied gained almost immediately, for example, from expulsion of intestinal worms, they would demand the drugs. Ancillary benefits such as expulsion of intestinal worms is not a new 


\section{RESEARCH ARTICLE}

observation and has been described as a possible means of improving compliance in a study of community-based treatment of onchocerciasis in Sierra Leone [19]. The observation that there will always be "wait and see attitude" individuals underscores the importance of having enough drug supplies, their availability over a defined, reasonable distribution period, reliable and accessible outlets as well as their perceived ancillary benefits. In the current study, our data suggest that one of the reasons for the greater awareness in the Com-DT/HS arm, maybe the active involvement in the preparatory process of people at the community level. All people who took part in the various group discussions in the Com-DT/HS mentioned that they had been informed about the drug distribution. The importance of involving the community has been noted in onchocerciasis control [20,21].

The finding that ComDT/HS arm was less clear about the purpose of the drugs and that it also reported more side effects than the HST arm, maybe explained by the difference in the medical orientation between the drug distributors in the two arms. Health workers in the HST arm would be more confident in emphasizing the purpose of the drugs and any side effects to be expected. Similarly, health workers would also tend to acknowledge more correctly any side effects and dismiss the minor ones leaving them unreported. In Phase I of this study, health workers had expressed their doubts in the community conducting duties of the medical profession successfully. However, during the evaluation of the current Phase, the benefits of involving the community appeared to outweigh this concern. Both the health workers and the community expressed appreciation of the community's contribution in drug distribution and even suggested the need to integrate other health services, for example, drugs for treatment of Schistosoma haematobium. As a health problem of high priority among communities receiving treatment for filariasis, integration of control of schistosomiasis with filariasis has been previously recommended [22] and its feasibility within the national PELF should be investigated.
In Kenya, diethylcarbamazine/albendazole combination is currently being used for filariasis elimination. This alternative strategy has welldocumented ancillary benefits and it can be expected that its use will not affect community compliance. In the present study, side effects were few, minor and could all be handled at the level of the dispensary. The safety of the coadministration of antifilarial drugs was confirmed in a review of 17 studies from multiple sites [23].

In this study, some of the communities residing in sparsely located homesteads indicated that provision of bicycles would have eased the CDDs duties. Yet others pointed out that provision of bicycles would be tantamount to undermining sustainability of the program since the communities would always look-up to the project for bicycle maintenance or divert the bicycles' use for personal and/or commercial gains. Drug delivery in PELF is not a year-long engagement and as long as the ComDT philosophy is upheld, using bicycles for personal and/or commercial gains to earn an income during the "off delivery periods" could turn out to be an innovative way for CDDs to earn an income, stay motivated and remain in the program for its life-span. Such observations emphasize the need for PELF to be sensitive and flexible enough to switch or combine different drug distribution strategies as maybe demanded by different situations. Similarly, salt fortified with DEC is an effective control strategy and was successfully used to eliminate lymphatic filariasis in China and several other countries [24].

In the neighbouring Tanzania, DECfortified salt has not only been shown to be effective in causing dramatic reductions in microfilariae, but also appear feasible for integration in large scale control programs based on community participation $[25,26]$. Hence, DEC medicated salt can and should be used as an additional synergy where applicable, for enhancing lymphatic filariasis elimination [27].

\section{Conclusion}

As shown, in Kenya, ComDT can be effectively implemented through the regular public health 


\section{RESEARCH ARTICLE}

service. In one round of drug distribution, ComDT achieved high levels of treatment coverage that appear adequate for filariasis elimination. To sustain it, some kind of incentive or compensation for the CDDs efforts was recognized as important. Although it should be noted that our observations are derived from one round of treatment only, this method of drug delivery can be recommended for PELF in Kenya and other similar sites. However, in view of the phasing out approach of PELFs, ComDT's need for organizational support, supervision and sustainability, other methods of drug delivery should still be explored, not for use in competition with the community drug delivery method, but to enhance it according to the varying needs of the different communities and sites. Drug distribution in the present study was designed for rural communities only. As there are clear socio-economic differences between rural and urban communities, a similar approach may not be as successful in urban settings. Therefore, there is need to investigate how high drug coverage levels can be achieved and sustained in large, urban areas.

\section{Acknowledgements}

We thank the staff of the Ministry of Health, especially both the DHMTs in Kilifi and Malindi districts, DFID-East Africa, GTZ-Kilifi District Development Program and KEMRI/Wellcome Trust Research Laboratories for their support. We acknowledge Phase 1 contributions of $\mathrm{Dr}$ Mary Amuyunzu-Nyamongo and the late Mr. Jacob Otieno Chiama, both formerly of KEMRI. We are indebted to the people of Kilifi and Malindi without whose co-operation this study would not have been successful. This investigation received financial support from the UNDP/World Bank/WHO Special Programme for Research and Training in Tropical Diseases and the work has been published with the permission of Director, KEMRI

\section{References}

1. Ottesen EA, Ismail MM, Horton J. The role of albendazole in programmes to eliminate lymphatic filariasis. Parasitology Today. 1999;15:382-386.
2. World Health Organization. Community directed treatment with ivermectin. Report of a multi-country study. Document TDR/AFR/RP/96.1. 1996.

3. Amazigo U, Noma M, Boatin BA, Etya'ale DE, Seketeli A, Dadzie KY. Delivery systems and cost recovery in Mectizan treatment for onchocerciasis. Annals of Tropical Medicine and Parasitology. 1998;92:S23-S31.

4. Roberts JM, Neumann E, Gockel CW, Highton RB. Onchocerciasis in Kenya 9, 11 and 18 years after elimination of the vector. Bulletin of the World Health Organization. 1967;37(2):195-212.

5. Wamae CN, Mwandawiro C, Wambayi E, Njenga S, Kiliku F. Lymphatic filariasis in Kenya since 1910, and the prospects for its elimination: a review. East African Medical Journal. 2001;78:595-603.

6. Central Bureau of Statistics. Population and housing census. Volume II. Population distribution by administrative areas and urban centres. Ministry of Finance and Planning, Republic of Kenya. 2001.

7. Estambale BBA, Simonsen PE, Knight R, Bwayo JJ. Bancroftian filariasis in Kwale District of Kenya. I. Clinical and parasitological survey in an endemic community. Annals of Tropical Medicine and Parasitology. 1994;88:145-151.

8. Mukoko DA, Pedersen EM, Masese NN, Estambale BB, Ouma JH. Bancroftian filariasis in 12 villages in Kwale district, Coast province, Kenya - variation in clinical and parasitological patterns. Annals of Tropical Medicine and Parasitology. Dec 2004;98(8):801-815.

9. Njenga SM, Wamae CN. Evaluation of ICT filariasis card test using whole capillary blood: comparison with Knott's concentration and counting chamber methods. Journal of Parasitology. 2001;87: 1140-1143.

10. Wamae CN, Gatika SM, Roberts JM, Lammie PJ. Wuchereria bancrofti in Kwale district, coastal Kenya: patterns of focal distribution of infection, clinical manisfestations and anti-filarial $\mathrm{IgG}$ 


\section{RESEARCH ARTICLE}

responsiveness. Parasitology. 1998; 116: 173-182.

11. World Health Organization. Communitydirected treatment of lymphatic filariasis in Africa. Report of a multi-centre study in Ghana and Kenya. Document TDR/IDE/RP/CDTI/00.2. 2000.

12. Plaisier AP, Subramanian S, Das PK, Souza $\mathrm{W}$, Lapa $\mathrm{T}$, Furtado AF, et al. The LYMFASIM simulation program for modelling lymphatic filariasis and its control. Methods of information in Medicine. 1998;37:97-108.

13. Stock R. Distance and the utilisation of health facilities in rural Nigeria. Social Science and Medicine. 1983;17:563-570.

14. Kipp W, Burnham G, Bamuhiiga J, Weis P, Buttner DW. Ivermectin distribution using community volunteers in Kabarole district, Uganda. Health Policy and Planning. 1998;13:167-173.

15. Mutabazi D, Duke BOL. Onchocerciasis control in Uganda: how can self-sustaining, community-based treatment with ivermectin be achieved? Annals of Tropical Medicine and Parasitology. 1998;92:195-903.

16. Askew I, Khan AR. Community participation in national family planning programs: some organisational issues. Studies in Family Planning. 1990;21:127142.

17. Gyapong M, Gyapong JO, Owusu-Banahene G. Community-directed treatment: the way forward to eliminating lymphatic filariasis as a public-health problem in Ghana. Annals of Tropical Medicine and Parasitology. 2001;95:77-86.

18. Katabarwa NM, Mutabazi D. The selection and validation of indicators for monitoring progress towards self-sustainment in community-directed, ivermectin-treatment programmes for onchocerciasis control in Uganda. Annals of Tropical Medicine and Parasitology. 1998;94:343-352.

19. Whitworth JA, Morgan D, Maude GH, Taylor DW. Community-based treatment with ivermectin. Lancet. 1988;2(8602):9798.

20. Akpala C, Okonkwo PO, Nwagbo D. Mobilization for mass distribution of ivermectin in Eastern Nigeria. Health Policy and Planning. 1993;8:78-81.

21. Katabarwa NM, Mutabazi D, Richards FO. Controlling onchocerciasis by communitydirected, ivermectin-treatment programmes in Uganda: why do some communities succeed and others fail? Annals of Tropical Medicine and Parasitology. 2000;94:343352.

22. Wamae CN, Mbugua GG. Integrated parasitic disease control program and the community role in sites with multiple species helminth infections. East African Medical Journal. 2000;77:631.

23. Horton J, Witt C, Ottesen EA, Lazdins JK, Addiss DG, Awadzi K, et al. An analysis of the safety of the single dose, two drug regimens used in programmes to eliminate lymphatic filariasis. Parasitology. 2000;121:S147-S160.

24. Lui J, Chen Z, Huang X, Zhaoping T. Mass treatment of filariasis using DEC-medicated salt. The Journal of Tropical Medicine and Hygiene. 1992;95:132-135.

25. Davis A, Bailey DR. The effect of salt medicated with diethylcarbamazine in bancroftian filariasis. Bulletin of the World Health Organization. 1969;41:195-208.

26. Meyrowitsch DW, Simonsen PE, Makunde WH. Mass diethylcarbamazine chemotherapy for control of bancroftian filariasis through community participation: comparative efficacy of a low monthly dose and medicated salt. Transactions of the Royal Society of Tropical Medicine and Hygiene. 1996;90:74-79.

27. Houston R. Salt fortified with diethycarbamazine (DEC) as an effective intervention for lymphatic filariasis, with lessons learned from salt iodization programmes. Parasitology. 2000;121:S161S173 


\section{RESEARCH ARTICLE}

Fig 1: Map of Kenya Showing lymphatic filariasis-endemic and suspect sites( Inset-areas of study: Kenya 1999)

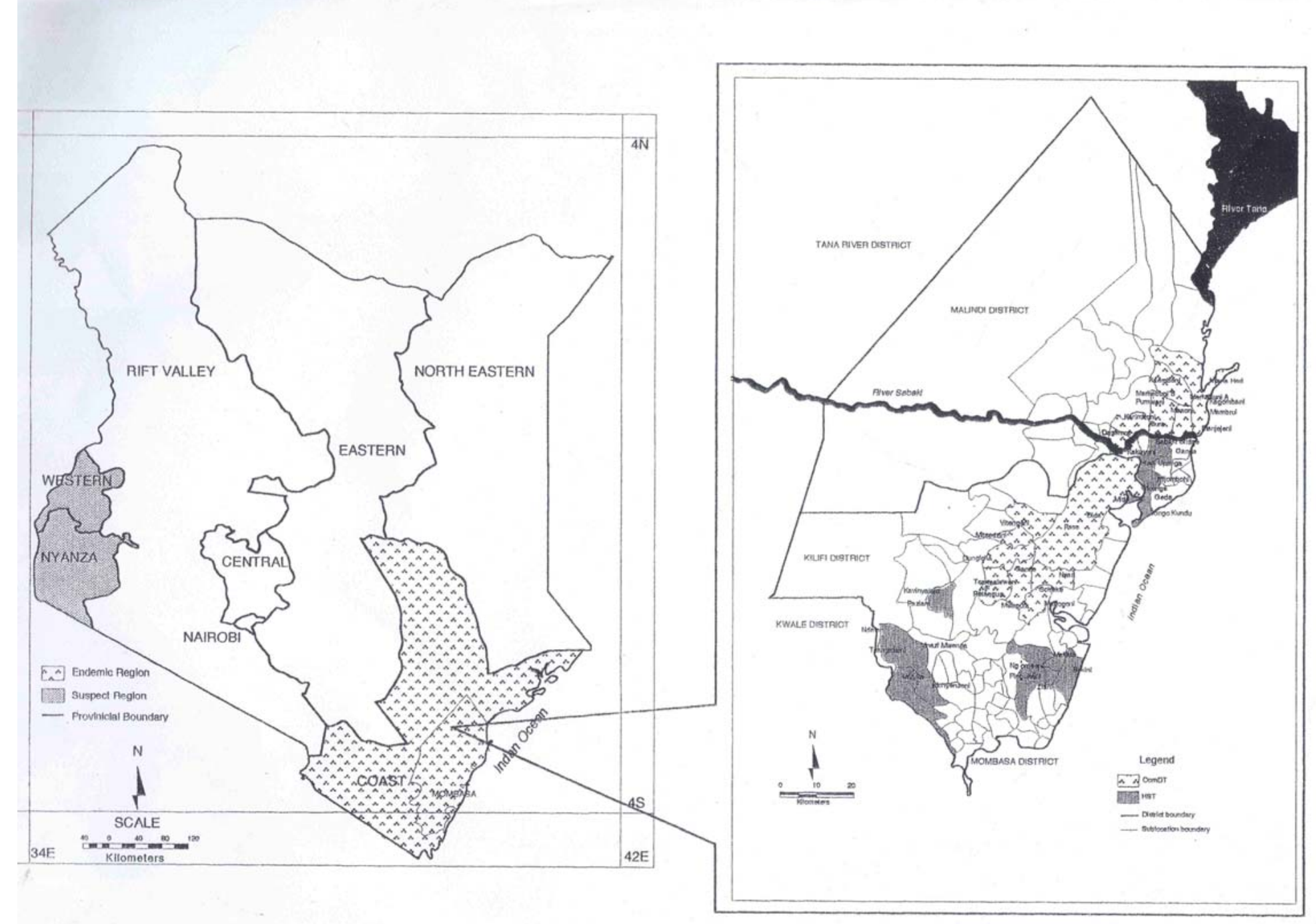

